\section{Preparation of Geological and Biological TEM Specimens by Embedding in Sulfur}

\author{
Richard C. Hugo and Sherry L. Cady \\ Portland State University \\ hugo@pdx.edu
}

\section{Introduction}

The technique of embedding TEM specimens in polymer resins for subsequent ultra-thin sectioning is well established [1]. For geological materials, this protocol prevents specimen damage introduced by ion beam thinning, and is often used for preparing friable or porous materials. However, traditional polymer-based embedding resins may introduce organic carbon contaminants, producing artifacts in carbon analyses. Thus, a carbon-free substitute for traditional embedding media is required to ensure accurate carbon analyses of embedded and ultramicrotomed TEM specimens.

A suitable technique that was first reported by Bradley [2] is to embed specimens in pure sulfur. In this technique, the specimen is immersed in liquid sulfur, the liquid is solidified, and the resulting block is ultramicrotomed as with traditional resins. Since sulfur has a high vapor pressure at room temperature, the ultra-thin sections are then placed in a separate vacuum chamber to sublimate the sulfur, yielding TEM specimens free of any embedding medium. This step prevents the introduction of undesirable sulfur vapor into the electron microscope column. Sulfur embedding has been used in investigations of carbon-bearing interplanetary dust particles [3] and meteorites [4], and has been used in a developmental technique for carbon isotope microanalysis [5]. Unfortunately, a systematic description of the procedure has not been published. We present our sulfur embedding protocol to disseminate this useful and difficult technique to a broader scientific community.

We find, as do others [7], that the greatest difficulty in utilizing this technique is solidifying a crack-free sulfur block. Another difficulty is handling the solidified block without fracturing or contaminating it. To properly solidify the block, the process temperature is controlled so that solidification proceeds slowly. To minimize handling and contamination problems, we solidify the block onto a rod designed to fit directly into the ultramicrotome chuck. By following optimum solidification and handling techniques, we produce ultrathin sulfur sections equal in quality to those produced from standard embedding resins.

We have applied this technique to analyses of both mineral and microbial specimens. For microbial specimens, the technique yields good results for characterizing carbon distribution, though it is not well suited for detailed images of cellular ultrastructure.

\section{Solidification Temperature and Block Size}

To properly solidify a sulfur block, the process temperature must be carefully controlled to optimize the solidification rate. Since sulfur readily supercools, solidification must be initiated by gently touching the liquid droplet with a sulfur crystal at the desired solidification temperature. During this operation the amount of solid in contact with the liquid droplet should be kept small enough that the liquid temperature is not lowered. If the solidification temperature is too low, the solidification front travels faster than liquid can be transported to the solid/liquid interface, resulting in microcracks as in Figure 1a. If solidification is initiated at the proper temperature, liquid convection outpaces the solid/liquid interface velocity, yielding a crack-free, nearly transparent solid block as in Figure $1 \mathrm{~b}$. If solidification is initiated at too high a temperature, the liquid droplet wicks onto the surface of the initiator crystal, where the liquid solidifies rapidly.
To determine the optimum solidification temperature, small sulfur particles were melted and then solidified at various temperatures. The speed of the solidification front was estimated by recording the time elapsed during solidification and dividing by the droplet diameter. Experiments took place on an aluminum block, with an embedded thermocouple, placed on a hot plate. The aluminum block surface was polished to a $1 \mu \mathrm{m}$ finish to prevent surface roughness from influencing the solidification process. Liquid droplets were allowed to cool to predetermined temperatures before initiating solidification as described above. As shown in Figure 2, solidification speeds increased dramatically below an initiation temperature of $90^{\circ} \mathrm{C}$, while extensive wicking of liquid sulfur onto the initiator crystal prevented proper solidification above $100^{\circ}$. Thus we found the optimum temperature range at which to initiate solidification to be $90-100^{\circ} \mathrm{C}$.

We often found that a cracked sulfur block could be repaired by partially remelting it and then slowly cooling to room temperature. This was performed by heating the defective block until it just began to melt, and then removing the block from the hot plate. In this solidification process, crystals remaining in the partial melt acted to initiate solidification as the droplet cooled, yielding a crack-free, polycrystalline block suitable for ultra-thin sectioning.

An additional parameter that must be controlled is the maximum temperature of the molten sulfur. As liquid sulfur is heated above $119^{\circ} \mathrm{C}$ it polymerizes, increasing its viscosity upon cooling so that the proper balance of solidification speed and convective mass transport cannot be achieved. Thus, droplets should be heated to just above the melting point.

The final parameter that must be controlled is the sulfur block size. Although any size sulfur droplet may conceivably be solidified without cracking if solidification proceeds within the optimum temperature range, we find that properly solidified blocks larger than $500 \mu \mathrm{m}$ fracture upon cooling as a result of shrinkage stresses. This $500 \mu \mathrm{m}$ size limit greatly restricts the size of samples that may be embedded in sulfur.

\section{Sulfur Ultramicrotomy}

The primary difficulty in ultramicrotomy of sulfur is its brittleness. This property renders it extremely sensitive to contaminant particles on the knife blade. Unfortunately, the brittle sulfur block itself provides a continuous supply of minute particles that adhere to the knife edge. Thus, the blade must be frequently and carefully cleaned with an eyelash probe. Sulfur's brittleness also makes a solidified block difficult to manipulate without fracturing. Thus, we do not trim sulfur blocks into a trapezoidal shape as is usual for polymeric blocks. Instead, we leave the block in its as-solidified, semi-spherical shape, and obtain circular ultra-thin sections. These circular sections do not adhere well to each other, making continuous ribbons of sections quite difficult to obtain.
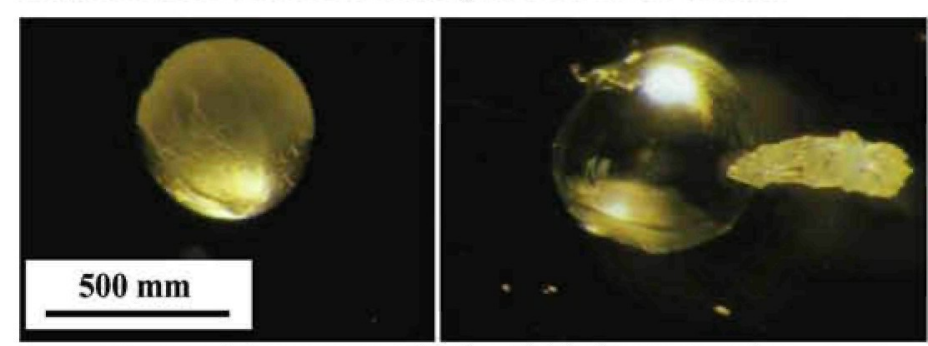

Figure 1 (A) A $450 \mathrm{~mm}$ S droplet solidified spontaneously at $\sim 40^{\circ} \mathrm{C}$. Solidification microcracks render the block translucent. (B) The same droplet, after remelting and manually nucleating solidification at $\sim 90^{\circ} \mathrm{C}$. This block is monocrystalline and crack-free. Note the initiator crystal welded to the block surface 


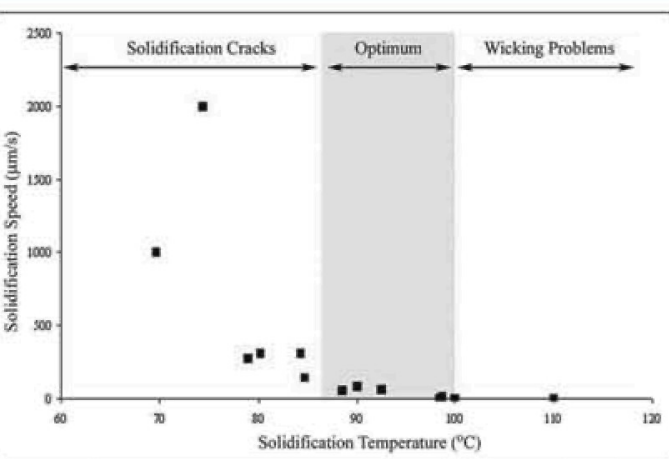

Figure 2. As solidification initiation temperature increases, the solidification speed and amount of solidification cracking decrease. The temperature range in which we prepared acceptable samples was $88-100{ }^{\circ} \mathrm{C}$

A second, albeit minor, challenge with sulfur is its variable refractive index. In standard ultramicrotomy of polymers, we estimate the thickness of ultra-thin sections by comparing their interference colors in the knife boat with a standard color-thickness chart. Since sulfur's refractive index is $\sim 2$, compared with $\sim 1.5$ for most embedding resins, sulfur interference colors shift towards longer wavelengths. Furthermore, sulfur is birefringent, causing the interference color of an ultrathin section to vary slightly according to the crystallographic orientation of the block. Although the color shift is always towards longer wavelengths, the precise extent of this shift is not known in practice since the block's crystallographic orientation is not controlled, and thickness measurements are therefore approximate.

To illustrate these issues in practice, we embedded a $100 \mu \mathrm{m}$ particle of cured EMBed 812 polymer resin in a $400 \mu \mathrm{m}$ sulfur droplet, solidified at $95^{\circ} \mathrm{C}$, and ultramicrotomed the resulting block, yielding ultra-thin sections containing both polymer and sulfur regions. As shown in Figure 3, ultra-thin sections that appeared gold in polymer regions were purple to blue in sulfur regions. Likewise, sections that appeared silver in polymer regions appeared pale to dark gold in sulfur. Further, we found the polymer regions to be quite smooth while the sulfur regions were scratched and wrinkled. These defects within the sulfur are moot as they disappear prior to TEM examination because, as stated above, the ultrathin sulfur is sublimated away in vacuum prior to insertion into the TEM.
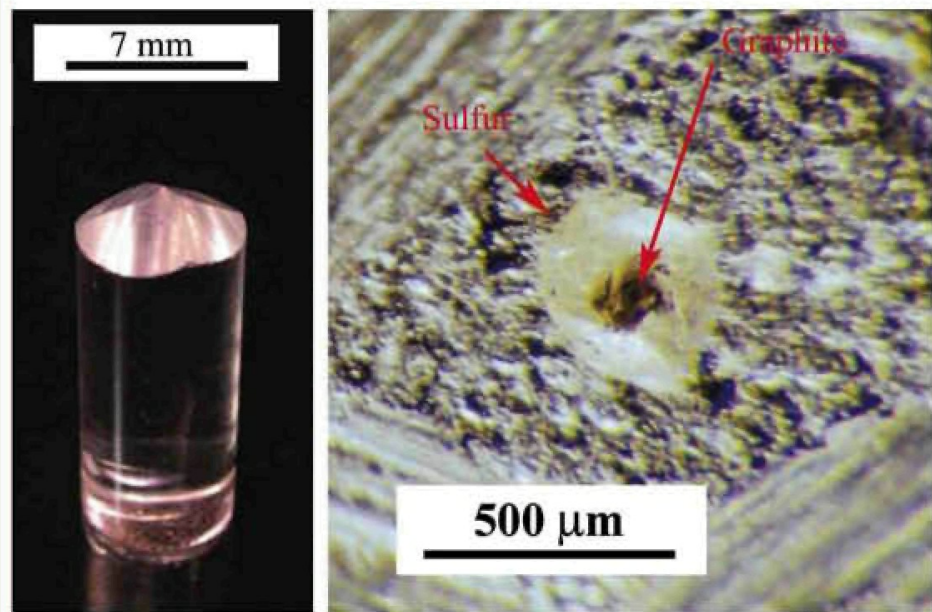

Figure 4. (A) A $7 \mathrm{~mm}$ glass stirring rod, cut to $20 \mathrm{~mm}$ length and tapered on a grinding wheel to a fine point. This point was then ground flat to provide $a \sim 1 \mathrm{~mm}^{2}$ work surface. (B) $A \sim 150 \mu$ m particle of graphite embedded in a crack-free sulfur block for ultramicrotoming.

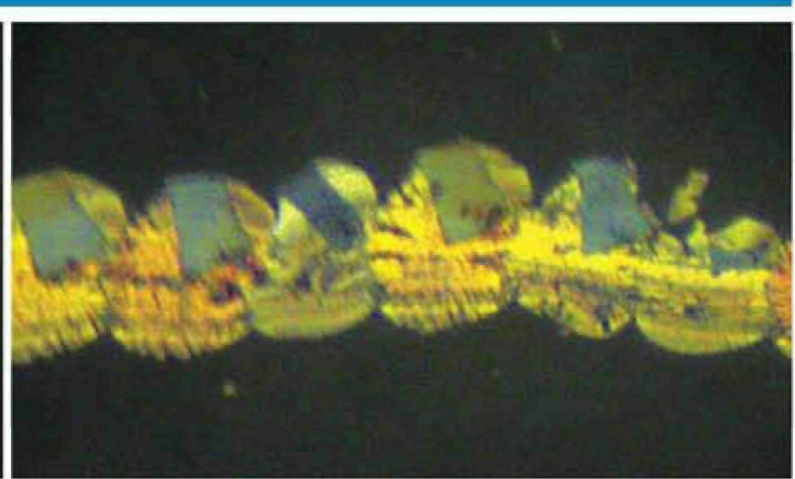

ptical images taken through ultramicrotome binoculars, showing ultra-thin sections floating in the knife boat. (A) A clump of sulfur/polymer sections in the $100-120 \mathrm{~nm}$ thickness range, as estimated by the dark gold color of the polymer regions of the sections. The sulfur regions are light blue to purple. (B) A 60-100 nm clump of sulfur/polymer sections. Polymer tions appear silver while sulfur regions appear pale gold to gold.

\section{Embedding Procedure}

1. Sulfur is sublimated by placing it on a hot plate at $\sim 140^{\circ} \mathrm{C}$. The vapor is collected on an inverted, acid-washed petri dish held over the sublimating liquid. The sulfur condensate consists of both solidified droplets and rod-shaped crystals used as solidification initiators.

2. A $400-500 \mu \mathrm{m}$ particle of sublimated sulfur is placed on an acid-washed glass slide and melted at $120^{\circ} \mathrm{C}$ on a hot plate. Meanwhile, a tapered glass or metal rod (as in Figure 4A) with $\sim 1 \mathrm{~mm}^{2}$ flat working surface, is also preheated to $120^{\circ} \mathrm{C}$ on the hot plate.

3. A $100-150 \mu \mathrm{m}$ particle to be embedded is placed upon the working surface of the heated tapered rod.

4. The slide with the molten sulfur droplet is inverted and the droplet is lowered onto the work surface to immerse the particle in liquid sulfur.

5. The work surface temperature is lowered to $90-95^{\circ} \mathrm{C}$ and held for several minutes to equilibrate.

6. A small rod-shaped sulfur crystal is brought to the liquid droplet surface to nucleate solidification. If performed gently enough, the solidification proceeds slowly and nearly invisibly, yielding a nearly transparent sulfur block as shown in Figure $4 \mathrm{~B}$. If the crystal is touched too forcefully to the liquid droplet, solidification proceeds suddenly, yielding a cracked block unsuitable for ultramicrotoming.

7. The tapered rod is fixtured into an ultramicrotome holder;

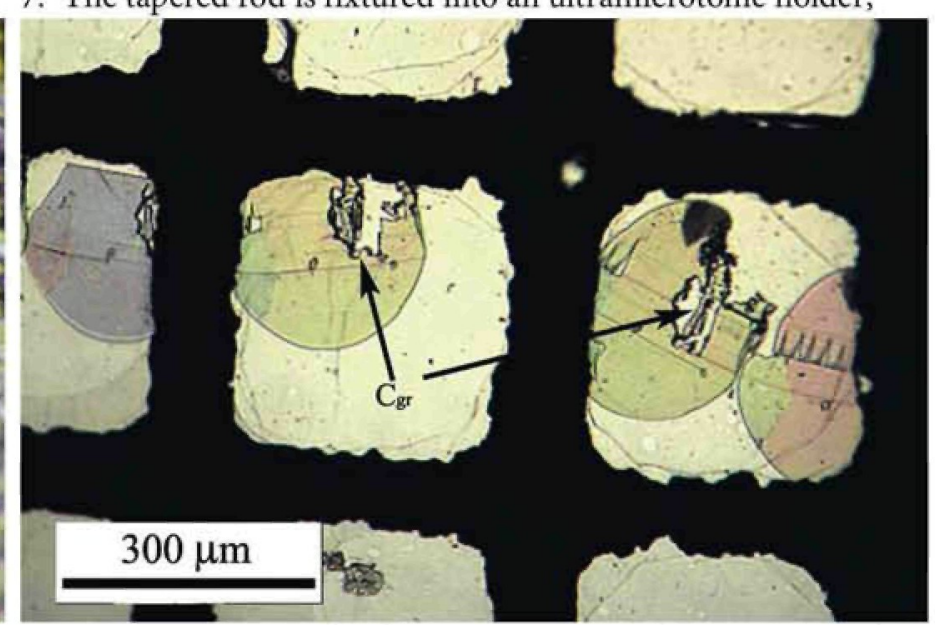

Figure 5. Optical light microscope image of a $\sim 100 \mathrm{~nm}$ thick ultramicrotomed sulfur sections on a lacey SiO support film. Graphite particles at arrows. Color variations between specimens due to thickness variations. However, note the color variations between different regions within each section, indicating that the sulfur block was a bicrystal. 
sections are cut and collected on a carbonfree support film, as shown in Figure 5.

8. The grid is placed under vacuum for an hour to sublimate the sulfur, yielding a TEM sample consisting of the ultramicrotomed particle free of any embedding medium.

\section{Results}

Sulfur-embedded Microbial Specimens:

As microbes become mineralized, it is of interest to examine the redistribution of elements, including carbon, within the cellular structure. Traditional biological specimen preparation methods utilize carbon-based materials to fix, dehydrate, embed and stain specimens. While the practice of sulfur embedding eliminates carbon contamination, it also bypasses steps that are necessary to preserve and delineate cellular components. To demonstrate the feasibility of sulfur embedding microbial samples, we prepared a TEM specimen of Chloroflexus auranticus, a filamentous bacterium that under some circumstances forms a tough sheath. It was hypothesized that this sheath would enable the organism to be successfully sulfur embedded and ultramicrotomed without excessive damage.

A thick Chloroflexus mat from culture was rinsed $3 \mathrm{x}$ in distilled water to remove growth medium and air dried on a clean microscope slide. A particle from the residue was sulfur embedded and ultramicrotomed as described above, and examined using a JEOL $100 \mathrm{CXII}$ TEM operated at $100 \mathrm{kV}$. Although most cells were ruptured beyond recognition, many cells were sufficiently intact to observe the sheath, cell wall and some internal structure as shown in Figure 6. Certainly, this image is not useful for detailed ultrastructural examination; however, the technique holds promise for experiments focused on describing the carbon distribution in mineralized specimens.

Note that we used no fixatives, dehydrants or stains to demonstrate this worst-case embedding scenario. However, we could have employed an $\mathrm{OsO}_{4}$ fixative before air drying, or carbon-free stains to better delineate ultrastructural details. In addition, mineralization would have acted as a de facto fixative, stabilizing cells during embedding and sectioning.

\section{Carbon EDS Analyses - Comparison of Sulfur embedded material with Polymer Resins}

In the TEM, electrons scattered from the incident beam at high angles may impinge upon nearby materials and produce $\mathrm{X}$ rays, yielding unwanted artifacts in analytical spectra. Thus, even if the embedding medium does not penetrate the specimen and therefore is not directly illuminated by the incident beam, polymer resins can contribute carbon and other elemental counts to an X-ray spectrum. To demonstrate this, we embedded two particles from a spectroscopically pure graphite rod, one in sulfur and the other in LR White resin. Ultramicrotomed sections were collected on $300 \#$ $\mathrm{Cu}$ TEM grids with $\mathrm{SiO}$ support films. EDS analysis was performed at $200 \mathrm{kV}$ on an FEI Tecnai F20 HRTEM with STEM/EDS capability. EDS spectra from both samples are shown in Figure 7. Both spectra

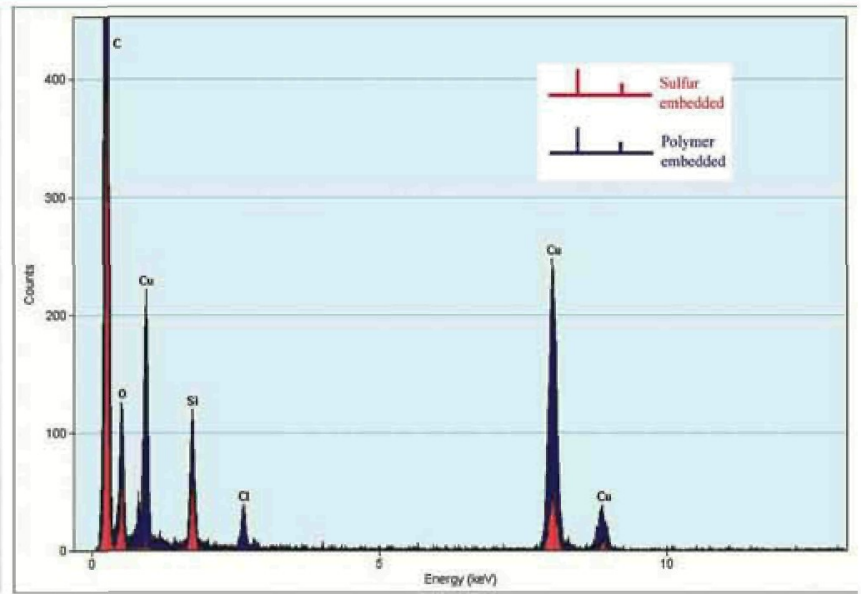

Figure 7. EDS spectra from sulfur embedded (red) and polymer embedded (blue) graphite particles. Note the artifact $\mathrm{Cl}$ peak in the polymer embedded specimen, which calls into question any quantitative analysis of the embedded material.

have artifact peaks from the Cu TEM grid and the SiO support film. The resin-embedded sample also has a significant $\mathrm{Cl}$ peak originating from the polymer. This implies that extraneous $\mathrm{C}$ counts also contributed to the spectrum by the resin. Note also the absence of a sulfur peak in the sulfur embedded spectrum, indicating that the sulfur has completely sublimated and contributes no signal to the EDS spectrum.

\section{Conclusions}

Although sulfur embedding presents several challenges compared to embedding in conventional polymer resins, it is an essential technique for accurately analyzing TEM specimens, whether geological or biological, for carbon content or distribution. To properly employ this technique, the user must carefully control the process temperature, and must also reduce specimens to the appropriate size. If the procedure is followed carefully, ultrathin sections may be produced that are not only free of any embedding medium but are also equal in quality to those embedded in conventional polymer resins.

\section{Acknowledgements}

The authors would like to acknowledge generous and helpful advice from Dr. D.J. Joswiak at the University of Washington. Dr. Y.M. Calvillo at Portland State University graciously supplied samples of cultured Chloroflexus auranticus. Analytical TEM data were obtained at the Multi-user Electron Microscopy Facility at Portland State University. This work was supported by the NASA Exobiology Program under grant NASA - NAG5-9579.

\section{References}

1. Bozzola, J.J. and Russell, L.D., Electron microscopy: principles and techniques for biologists. 2nd ed. 1999, Sudbury, Mass.: Jones and Bartlett.

2. Bradley, J.P., Keller, L., Thomas, K.L., Vander Wood, T.B., and Brownlee, D.E. (1993). Carbon analyses of IDPs sectioned in sulfur and supported on beryllium films, in Twenty-fourth lunar and planetary science conference, NASA Lunar and Planetary Science Conference.

3. Keller, L.P., Thomas, K.L., and McKay, D.S., Carbon petrography and the chemical state of carbon and nitrogen in IDPs, in Twenty-seventh Lunar and planetary science conference. 1996, Lunar and Planetary Science Conference. p. 659-660.

4. Nakamura, K., Zolensky, M.E.T.S., and Tomeoka, K. (2002). Raman spectroscopy of carbonaceous globules in the Tagish Lake chondrite, in 65th annual meeting of the Meteoritical Society, Meteoritical Society.

5. Hode, T., Kristiansson, P., Hugo, R.C., and Cady, S.L. (2004). A new non-destruc tive micro-analytical method for stable carbon isotope analysis of TEM-sections, in Third Astrobiology Science Conference. NASA AMES Research Center.

6. Joswiak, D.J. (2003) Private Communication. 


\section{It's the same with}

\section{microanalysis...}

\section{you want your \\ supplier to always \\ be there for you.}

At EDAX, we take enormous pride in providing today's best microcharacterization technologies. But this is not the end. In fact, installing your system is just the beginning of your relationship with EDAX.

We are equally proud of our unparalleled global support for all our customers. EDAX provides frequent, in-depth training classes, skilled service engineers on call to assist you and scientists to advise you in your specific applications.

Next time you are in the market for X-ray microanalysis, EBSD or micro-XRF systems, come to EDAX. We will not let go of you after the sale is made.

Discover what good service and support are all about at: www.edax.com/thinkEDAX

\section{EDAX

